ISSN 0103-5150

Fisioter. Mov., Curitiba, v. 26, n. 1, p. 63-70, jan./mar. 2013

Licenciado sob uma Licença Creative Commons

\title{
Caracterização da qualidade de vida de adolescentes com escoliose idiopática
}

\author{
Characterizing the quality of life of \\ adolescents with idiopathic scoliosis
}

\section{Giselle Cristina Lopes Rosanova ${ }^{[a]}$, Paula Maria Ferreira Camarini ${ }^{[b]}$, Bruna Sayuri Gabriel ${ }^{[c]}$, Anamaria Siriani de Oliveira ${ }^{[\mathrm{d}]}$}

[a] Fisioterapeuta, especialista em Fisioterapia Pediátrica pela Universidade de São Paulo (USP), aperfeiçoanda da Associação de Assistência à Criança com Deficiência (AACD), São Paulo, SP - Brasil, e-mail: giselle.rosanova@gmail.com

[b] Fisioterapeuta, especialista em Fisioterapia Musculoesquelética, Irmandade da Santa Casa de Misericórdia de São Paulo (ISCMSP), Departamento de Fisioterapia, Ribeirão Preto, SP - Brasil, e-mail: paula_camarini@yahoo.com.br

[c] Fisioterapeuta com Aprimoramento em Ortopedia e Traumatologia pelo Hospital das Clínicas de Ribeirão Preto da Universidade de São Paulo, Ribeirão Preto, SP - Brasil, e-mail: bruninha_sayuri@yahoo.com.br

[d] Fisioterapeuta, docente da Universidade de São Paulo de Ribeirão Preto, Ribeirão Preto, SP - Brasil, e-mail: siriani@fmrp.usp.br

\section{Resumo}

Introdução: Pacientes com escoliose possuem autoestima mais baixa, pior percepção corporal, dores na coluna vertebral; sentem-se menos saudáveis e mais infelizes que indivíduos sem deformidades na coluna. Objetivo: Avaliar a qualidade de vida (QV) de adolescentes com escoliose idiopática (EI) por meio do questionário autoadministrável Br-SRS-22r. Materiais e métodos: Trata-se de estudo transversal. Foram selecionados por conveniência 30 pacientes com EI, média de idade de 15,5 $( \pm 2,6)$ anos e curvaturas de valores médios de 28,4 ( $\pm 21,0)$ graus Cobb. A estatística descritiva apresentada mostra a distribuição de frequências dos escores dos cinco domínios do Br-SRS-22r. Resultados: Analisando-se o domínio função, observa-se que 93,3\% dos voluntários apresentaram escore acima de 3. Para o domínio autoimagem, nota-se maior distribuição dos pontos, sendo que $26,7 \%$ pontuaram entre 2 e 2,9. A distribuição dos escores dor e saúde mental foi semelhante, observando-se maior concentração entre 3 e 3,9 pontos (36,7 e 40\%, respectivamente). No domínio satisfação com o tratamento, 78,6\% dos voluntários apresentaram escore acima de 4. Conclusão: Os achados deste estudo para os domínios dor, função física e autoimagem corroboram estudos anteriores. Os resultados demonstraram que, em uma amostra de adolescentes com EI cuja maioria é tratada conservadoramente 
apenas por medidas fisioterapêuticas, o impacto da condição na percepção da QV foi considerado mediano, nos domínios dor, aparência e saúde mental, a fraco, no domínio função física.

Palavras-chave: Escoliose. Questionário. Qualidade de vida.

\section{Abstract}

Introduction: Idiopathic scoliosis (IS) patients have poor self-esteem and self-image/appearance perception, more spine pain and feel less healthy and less happy than individuals without spinal deformities. Objective: To evaluate the quality of life (QOL) of IS adolescents using the Br-SRS-22r self-administrated questionnaire. Materials and methods: This is a cross-sectional study. Thirty IS patients, mean age of 15.5 ( \pm 2.6$)$ years old and mean curvature magnitude of $28.4( \pm 21.0)$ Cobb degrees were selected. Descriptive statistics presented the frequency distribution scores of each Br-SRS-22r domain. Results: In the function domain, it was observed that $93.3 \%$ of the volunteers had a score above 3. For the self-image domain, there was a greater distribution of points in which $26.7 \%$ scored between 2 and 2.9. The distribution of the pain and mental health scores was similar, with a higher concentration between three and 3.9 points (36.7 and 40\%, respectively). In the treatment satisfaction domain, 78.6\% of volunteers scored above 4. Conclusion: Findings for pain, physical functional and self-appearance domains were in agreement with those previous published. Presented results demonstrated that, in a sample composed mainly by IS patients being conservatively treat with physical therapy, the condition impact was mild, in pain, appearance and mental health domains, to weak in physical function domain.

Keywords: Scoliosis. Questionnaire. Quality of life.

\section{Introdução}

A qualidade de vida $(\mathrm{QV})$ refere-se ao bem-estar físico, emocional e psicológico das pessoas e à sua capacidade de executar as tarefas diárias $(1,2)$. Em 1995, o grupo World Health Organization Quality of Life (WHOQOL) definiu qualidade vida como a percepção do indivíduo sobre sua posição na vida, no contexto da cultura e sistemas de valores em que vive e em relação a seus objetivos, expectativas, padrões e preocupações $(1,2)$. Recentemente, o interesse em avaliar a QV está aumentando entre os pesquisadores e clínicos, e essa área está em crescente evolução (3-8). A maioria dos tratamentos realizados em ortopedia não afeta a expectativa de vida, mas a sua qualidade $(4,9)$. As medidas tradicionais objetivas como amplitude de movimento, força muscular e radiografia são de grande utilidade, porém não proporcionam uma avaliação satisfatória dos aspectos funcionais, sociais e psicológicos da saúde mais relevantes ao paciente. Por isso, nos últimos anos, o desenvolvimento de avaliações realizadas pelos pacientes sobre sua percepção a respeito de sua condição tem adicionado outra dimensão para a análise dos resultados clínicos $(5,9,10,11)$.

As pesquisas qualitativas, com medidas subjetivas, podem auxiliar na escolha de um tratamento e informar sobre os possíveis efeitos da intervenção $(1,3)$. Além disso, a avaliação é feita pela perspectiva do indivíduo. Dessa maneira, as medidas subjetivas tornam-se um complemento importante para a avaliação tradicional e faz com que não só as preocupações do profissional da saúde sejam consideradas, mas também as do paciente $(4,9)$.

Uma importante ferramenta utilizada para a avaliação da QV é o questionário autoadministrativo Scoliosis Research Society-22 (SRS-22r), destinado a portadores de escoliose idiopática $(12,13)$, cuja versão brasileira (Br-SRS-22r) foi validada em 2010 (14). 0 Br-SRS-22r é constituído de 22 questões distribuídas em cinco domínios: autoimagem/aparência (AA); função/atividade (FA); saúde mental (SM); dor (D) e satisfação com o tratamento (ST), e seus escores variam de 1 a 5 , sendo 5 a melhor condição $(12,13,14)$.

A escoliose pode ser definida como uma deformidade tridimensional da coluna, com desvio das curvaturas fisiológicas no plano sagital e frontal, normalmente associada à rotação vertebral no plano transversal (15). Pode estar associada à má formação de vértebras, fraturas e deslocamento da espinha, diferença de membros inferiores, desequilíbrio hormonal, má postura e espasmos musculares $(16,17)$. Quando não está associada a esses fatores, é chamada 
escoliose idiopática, que corresponde à maioria dos casos $(16,17)$. A EI afeta similarmente pessoas do sexo feminino e masculino (18) antes da maturidade sexual (cerca de $3 \%$ dos adolescentes), entretanto, a progressão é mais frequente e mais severa em pessoas do sexo feminino $(18,19)$. Essa afecção pode acarretar vários problemas físicos, como dor nas costas, prejuízo pulmonar, diminuição da mobilidade e da habilidade para o trabalho, bem como problemas psicossociais tais como a preocupação com a aparência e com o prognóstico incerto (20).

Estudos de QV que utilizaram diferentes instrumentos para essa avaliação subjetiva, como o Berner Questionnaire for Well-Being (20), por exemplo, mostram que pacientes com escoliose possuem autoestima mais baixa, pior percepção corporal, dor na coluna vertebral, sentem-se menos saudáveis e mais infelizes que indivíduos sem deformidades na coluna (20, 21, 22). Payne et al. (23) mostraram que, entre adolescentes, a deformidade pode representar maior risco de pensamentos suicidas e preocupação com o desenvolvimento corporal. Além disso, outros estudos constataram que a presença dessa patologia é responsável por dificuldades para participar de atividades físicas e sociais, restringindo as atividades de vida diárias $(22,23,24,25)$.

Parent et al. (19) também relatam a hipótese de que fatores como idade, tipo de tratamento, ângulo de Cobb e tipo de curva podem influenciar o impacto da escoliose idiopática na QV. Entretanto, Danielsson et al. (26) relatam, em um estudo comparativo entre adolescentes tratados com colete e os não tratados, que, na vida adulta, não houve diferença na QV de ambos os grupos.

Durante pesquisa realizada nas principais bases de dados, poucos artigos foram encontrados com dados referentes à QV em brasileiros com EI. Portanto, levando-se em conta o impacto na QV desses adolescentes encontrado nos estudos citados e a falta de estudos sobre a população brasileira, torna-se clara a importância de avaliar a QV desses indivíduos. Assim, o objetivo desta pesquisa é analisar a QV de adolescentes com escoliose idiopática por meio da utilização do questionário Br-SRS-22r.

\section{Materiais e métodos}

\section{Aspectos éticos}

Todos os voluntários incluídos e seus responsáveis foram esclarecidos a respeito do procedimento do estudo em uma entrevista prévia e assinaram um termo formal de consentimento livre e esclarecido para a participação no estudo. 0 termo foi elaborado segundo a Resolução n. 196, de 10 de outubro de 1996, do Conselho Nacional da Saúde e aprovado pelo Comitê de Ética em Pesquisa envolvendo Seres Humanos do HC-FMRP, processo HCRP n. 9853/2005.

\section{Voluntários}

Trata-se de um estudo de corte transversal, conduzido num período de sete meses, de fevereiro a setembro de 2010, no qual foram incluídos consecutivamente, por ordem de comparecimento em consultas médicas ortopédicas ou de fisioterapia, indivíduos diagnosticados como portadores de escoliose idiopática do adolescente, com idade acima de 12 anos (idade mínima para entendimento do questionário) e ângulo de Cobb maior ou igual a $11^{\circ}$ (angulação mínima para levar a algum impacto na qualidade de vida) $(27,28)$. Assim, foram incluídos 30 pacientes do setor de ortopedia de um serviço público de saúde de nível terciário e de um serviço para pacientes conveniados de nível secundário, sendo 25 do sexo feminino e 5 do sexo masculino. A média de idade da amostra foi de $15,5( \pm 2,6)$ anos, apresentando curvaturas de valores médios de 28,4 $( \pm 21,0)$ graus Cobb, variando entre $11^{\circ}$ e $70^{\circ}$. 0 ângulo de Cobb foi calculado a partir da imagem radiográfica mais recente disponível, como realizado no estudo de Morrissy et al. (29).

Diferentes níveis de comprometimento foram incluídos para compor uma amostra capaz de caracterizar diferentes níveis de repercussão da EI sobre a $\mathrm{QV}$, considerando pacientes tão distintos quanto os acompanhados apenas para tratamento conservador ou aqueles em período pós-cirúrgico tardio, havendo também voluntários com curvas ainda não estabilizadas. Essa amostra foi composta por dois pacientes pós-cirúrgicos, dois pacientes sem tratamento, cinco fazendo tratamento apenas com brace e os demais (63\% da amostra) são pacientes com curvaturas leves que seguem exclusivamente tratamentos por exercícios. Foram critérios de exclusão o analfabetismo ou a falta de capacidade cognitiva para interpretação das perguntas do questionário, já que ele se propõe ser autoadministrado, e possuir diagnóstico relatado de doenças neurológicas, reumáticas ou outras condições dolorosas crônicas. 
Procedimento

O questionário Br-SRS-22r foi apresentado aos voluntários sempre pelo mesmo avaliador que os instruiu a respondê-los, sem auxílio dos acompanhantes, já que a versão brasileira foi idealizada para ser autoadministrável (14). 0 examinador foi um membro externo à equipe que procedeu o diagnóstico ou que acompanhava o tratamento para reduzir a influência do examinador, em especial, na reposta sobre a satisfação com o tratamento.

Os pacientes selecionados para o estudo responderam ao questionário em um consultório, antes de passarem em consulta médica de rotina. Não registramos perdas amostrais por preenchimento incompleto ou falta de compreensão das questões. Os voluntários levaram cerca de 20 minutos para completar o preenchimento do questionário.

\section{Análise dos dados e estatística}

Cada um dos itens do Br-SRS-22r é pontuado de 1 a 5, sendo 5 a melhor condição de saúde e 1 o menor comprometimento da QV no item específico. Suas 22 questões estão distribuídas nos domínios autoimagem/aparência (AA - cinco questões); função/atividade (FA - cinco questões); saúde mental (SM - cinco questões); dor (D - cinco questões); e satisfação com o tratamento (ST - duas questões). 0 maior escore total possível é 110 pontos e, quanto mais próximo desse valor, melhor a percepção individual sobre sua qualidade de vida.

O escore (ou pontuação) de cada domínio foi obtido do valor médio da pontuação das questões que o compõem, ou seja, somando o escore das questões correspondentes ao domínio de dividindo esse resultado pelo número de questões respondidas (12). Como este estudo possui característica descritiva e não há teste de hipóteses, a análise apresentada baseia-se em estatística descritiva e na distribuição de frequências dos escores em cada um dos domínios do Br-SRS-22r.

\section{Resultados}

No Quadro 1 são apresentadas as porcentagens médias de escolha dos escores atribuídos a cada um dos domínios do questionário Br-SRS-22r. Dois candidatos não responderam às questões do domínio satisfação com o tratamento porque, embora diagnosticados, ainda não haviam iniciado nenhum tipo de tratamento. No entanto, dos 28 que responderam a esse item, 78,6\% dos voluntários apresentaram escore acima de 4 para esse domínio; isso significa que, em média, esses voluntários, embora tenham características heterogêneas e sejam submetidos a diferentes tratamentos, apresentavam relativa satisfação com seus tratamentos.

Com relação ao domínio função física/atividade, observou-se que 93,3\% dos 30 voluntários avaliados apresentaram pontuação maior que 3. Considerando o escore 1 como a pior percepção de impacto da condição na qualidade de vida relacionada ao item e 5 a melhor, e que as respostas se concentram entre os escores $4 \mathrm{e}$ $5(46,7 \%)$, isso significa que, em média, os voluntários apresentam uma percepção relativamente não negativa do impacto da EI em suas funções físicas. Já para o domínio autoimagem, pode-se observar que, apesar de a maioria dos escores também estar concentrada acima de $3(66,7 \%)$, nota-se que $26,7 \%$ pontuaram entre 2 e 2,9 e nenhum voluntário obteve escore 5 .

A distribuição dos escores dor e saúde mental foi semelhante, observando-se maior concentração entre 3 e 3,9 pontos (36,7 e 40\%, respectivamente) e apenas $16,7 \%$ dos voluntários pontuaram abaixo de 3 nesses dois domínios.

O Gráfico 1 mostra a distribuição da pontuação média de cada um dos cinco domínios em valores absolutos para os 30 voluntários avaliados nos domínios função física/atividade, dor, autoimagem e saúde mental, e para os 28 que preencheram o domínio satisfação com o tratamento. Embora se trate de uma amostra relativamente pequena e heterogênea, é possível notar que, independente do domínio avaliado, as medianas estão sempre acima da pontuação 3 , indicando uma percepção de qualidade de vida variando de média a satisfatória.

\section{Discussão}

\section{Domínio função/atividade}

Pode-se observar que o domínio função/atividade apresentou $46,7 \%$ das respostas concentradas no escore entre 4 e 4,9. Resultado semelhante foi encontrado no estudo de Cheung et al. (30), que comparou a QV de adolescentes com EI apenas em observação 
Quadro 1 - Porcentagem de voluntários por faixa de escores em cada um dos domínios do Br-SRS-22r ( $\mathrm{n}=30$ )

\begin{tabular}{lcccccc}
\hline & \multicolumn{7}{c}{ Escore } \\
\cline { 2 - 7 } Domínio & $\mathbf{1 - 1 , 9}$ & $\mathbf{2 - 2 , 9}$ & $\mathbf{3 - 3 , 9}$ & $\mathbf{4 - 4 , 9}$ & $\mathbf{5}$ & SR* $^{*}$ \\
\hline Função/atividade & $3,3 \%$ & $3,3 \%$ & $36,7 \%$ & $\mathbf{4 6 , 7} \%$ & $10 \%$ & 0 \\
Dor & $3,4 \%$ & $13,3 \%$ & $\mathbf{3 6 , 7 \%}$ & $23,3 \%$ & $23,3 \%$ & 0 \\
Autoimagem/aparência & $6,7 \%$ & $26,7 \%$ & $\mathbf{4 6 , 7 \%}$ & $20 \%$ & 0 & 0 \\
Saúde mental & $3,3 \%$ & $13,3 \%$ & $\mathbf{4 0 \%}$ & $36,7 \%$ & $6,7 \%$ & 0 \\
Satisfação com o tratamento & $3,3 \%$ & $10 \%$ & $6,7 \%$ & $\mathbf{4 0 \%}$ & $33,3 \%$ & $6,7 \%$ \\
\hline
\end{tabular}

Legenda: ${ }^{\star}=$ sem resposta.

Fonte: Dados da pesquisa.

Nota: As porcentagens em negrito indicam a faixa de escore com a maior porcentagem de voluntários.

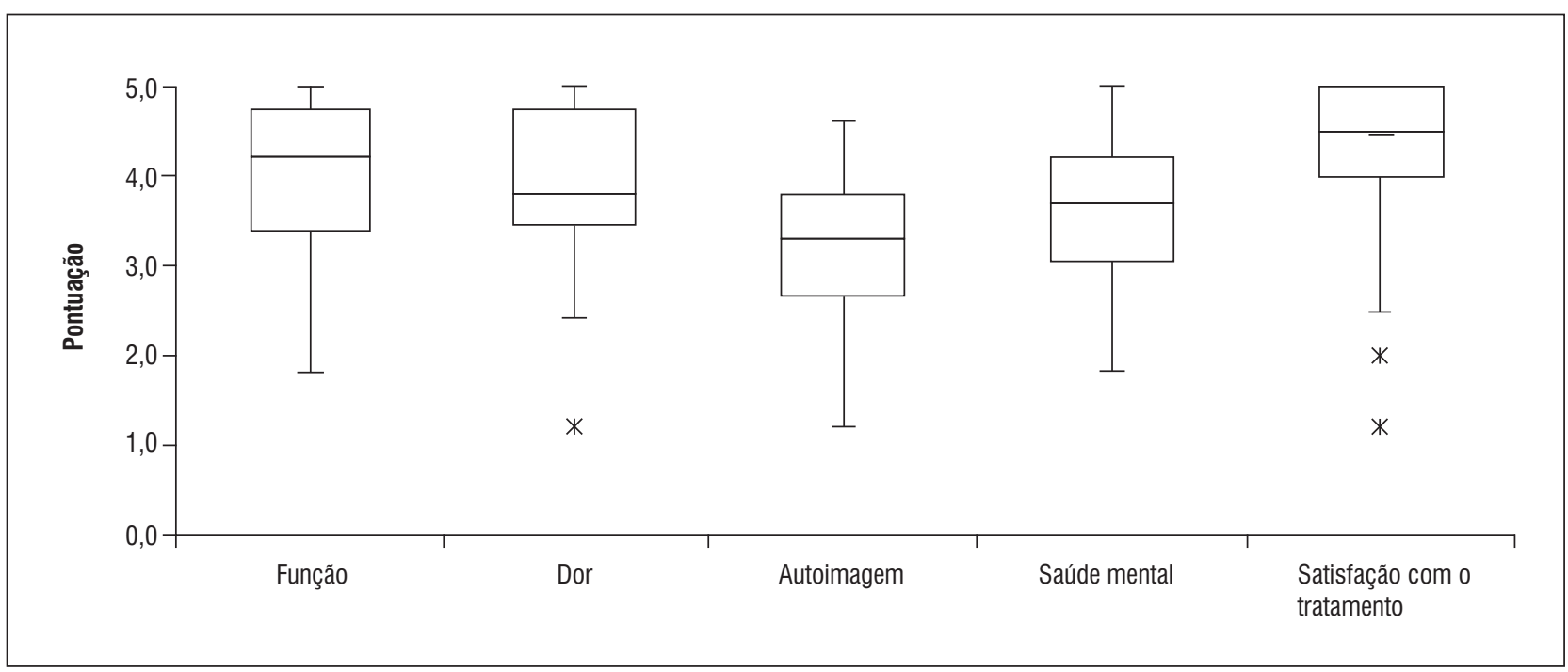

Gráfico 1 - Distribuição da pontuação média de cada um dos cinco domínios

Legenda: 0s boxplots apresentam os dados de mediana dos escores do Br-SRS-22r (linha interna da caixa). As caixas representam $1^{\circ}$ e $3^{\circ}$ quartis. As barras indicam os valores máximos e mínimos dos escores obtidos. Os asteriscos marcam dados considerados "outliers".

Fonte: Dados da pesquisa.

Nota: Os valores dos domínios função, dor, autoimagem e saúde mental foram calculados a partir das respostas da amostra total $(\mathrm{n}=$ 30). Os valores do domínio satisfação com o tratamento foram calculados sem os dados de dois voluntários que não estavam se submetendo a nenhum tratamento no momento da entrevista $(n=28)$.

(nenhum tratamento) com pacientes que utilizaram colete como forma de tratamento por meio do SRS22. Neste trabalho, os escores encontrados indicam uma percepção de função relativamente boa e podem estar relacionados à média do ângulo de Cobb, que foi de $28,4^{\circ}( \pm 21,0)$. No entanto, Cheung et al. (30) não encontraram escores diferentes de percepção de qualidade de vida relativa à função física e atividades quando dividiram os achados de seus voluntários em subgrupos de acordo com o ângulo de Cobb $\left(<20^{\circ}\right.$ $\mathrm{n}=9 ; 20<30^{\circ} \mathrm{n}=20 ; 30<40^{\circ} \mathrm{n}=20$ e $>40^{\circ} \mathrm{n}=7$ ), encontrando pontuações média entre 4 e 4,9 para todos os subgrupos. Assim, é possível que valores de inclinação lateral entre 20 e $40^{\circ}$ influenciem pouco a autopercepção dos portadores de EI em relação às funções físicas e atividades questionadas pelo SRS-22.

\section{Domínio dor}

Freidel et al. (20), Goldberg et al. (21), Mayo et al. (22) e relatam impacto na QV de pacientes 
com escoliose idiopática em virtude da dor. Neste estudo, os valores médios da pontuação deste domínio concentraram-se entre 3 e 3,9, ou seja, no centro da escala. No entanto, em outros estudos, o domínio dor apresentou escores entre 4 e 4,9 (26, $30,31)$, demonstrando impacto da dor na percepção da QV dos pacientes ainda menor, mesmo quando eles estão em tratamento conservador, usando braces, como no caso do estudo de Cheung et al. (30). Como nossos voluntários possuem menores valores de ângulo de Cobb e, em geral, estão apenas realizando tratamento fisioterapêutico, essa diferença, com relativo maior impacto da dor na qualidade de vida dos amostrados deste estudo, pode se dar por diferenças culturais da população brasileira e pela diferença na forma de lidar com a dor em diferentes países (32).

\section{Domínio autoimagem/aparência}

A escoliose pode levar a assimetrias do tronco relacionadas à magnitude do ângulo de Cobb e, assim, afetar a percepção da aparência dos portadores (23). 0 domínio autoimagem/aparência apresentou $46,7 \%$ dos escores concentrados entre 3 e 3,9, faixa de pontuação que corresponde ao valor central da ordem numérica proposta para escalonar a percepção dos voluntários. Valores semelhantes também foram encontrados nos trabalhos de Danielsson et al. (24), Cheung et al. (30) e Wang et al. (31). A autoimagem tem se demonstrado fator de grande impacto na QV dos pacientes com escoliose idiopática em razão da deformidade aparente, especialmente em adolescentes, fase em que há uma grande preocupação com a imagem (23). No entanto, neste estudo, esse impacto mediano pode ser justificado pelo valor médio dos ângulos de Cobb, que foi menor que $30^{\circ}$ - valor considerado relativamente pequeno, visto que na faixa entre 10 e $25^{\circ}$ Cobb, os pacientes, frequentemente, são portadores de deformidades leves e têm apenas a indicação de exercícios como tratamento conservador (33).

\section{Domínio saúde mental}

Payne et al. (23) relatam risco aumentado de pensamentos suicidas, maior ocorrência de alcoolismo e preocupação com o desenvolvimento corporal em adolescentes com escoliose idiopática, o que representa grande impacto da escoliose quando se trata de saúde mental. Neste estudo, $40 \%$ dos voluntários apresentaram pontuação entre 3 e 3,9 para este domínio. Esse valor difere daqueles encontrados em outros estudos recentes, que tiveram os valores maiores que 4 e, portanto, indicam melhor condição nas questões relativas à saúde mental $(26,30,31)$. Como os voluntários deste estudo têm deformidades consideradas leves e seguem, a maioria, apenas tratamento conservador sem o brace, tal discrepância pode ser explicada em razão da valorização da imagem corporal na cultura brasileira, capaz de influenciar a saúde mental desses adolescentes.

\section{Domínio satisfação com o tratamento}

Danielsson et al. (24), Cheung et al. (30) e Wang et al. (31), em estudos anteriores, relataram pontuação média entre 3 e 3,9 para o domínio satisfação com o tratamento. Este estudo, por outro lado, apresentou valores maiores de satisfação com o tratamento, com cerca de $79 \%$ da pontuação média acima de 4. É importante ressaltar que essa diferença entre os resultados pode ser explicada pelas características das amostras envolvidas nos estudos. No nosso, dois voluntários, dos 30 que participaram, ainda não estavam em tratamento, seis deles faziam uso de braces e outros dois já haviam sido submetidos à cirurgia de correção. Os estudos de Danielsson et al. (24), Cheung et al. (30) e Wang et al. (31) envolveram pacientes tratados cirurgicamente ou com braces, ao contrário de nossa amostra, composta em geral por pacientes em tratamento conservador, apenas por medidas fisioterapêuticas.

\section{Conclusão}

Os resultados demonstraram que, nos pacientes avaliados, o impacto da condição na percepção da QV foi considerado como mediano nos domínios dor, aparência e saúde mental, fraco, no domínio função física. Este estudo apresenta uma amostra relativamente pequena e de adolescentes apenas de uma cidade do país. Considerando-se a grande diversidade cultural encontrada no Brasil, sugerimos um estudo com maior número de voluntários de diversas regiões para esclarecer os achados relacionados à dor e saúde mental. 


\section{Referências}

1. Donald A. What is quality of life? Clinical Lecturer in Epidemiology. London: University College; 2003;1:1-6.

2. Skevington SM, Sartorius N, Amir M, Whoqol-Group. Developing methods for assessing quality of life in different cultural settings: the history of the WHOQOL instruments. Soc Psychiatr Psychiatr Epidemiol. 2004;39:1-8. doi:10.1007/s00127-004-0700-5.

3. Campos CC, Manzano GM, de Andrade LB, Castelo A Filho, Nóbrega JAM. Tradução e validação do Questionário de avaliação de gravidade dos sintomas e do estado funcional na Síndrome do Túnel do Carpo. Arq Neuropsiquiatr. 2003;61(1):51-5. doi:10.1590/ S0004-282X2003000100009.

4. Padua R, Padua L, Ceccarelli E, Romanini E, Bondy $\mathrm{R}$, Zanoli G, et al. Cross-cultural adaptation of the lumbar North American Spine Society questionnaire for the italian-speaking patients with lumbar spinal disease. Spine 2001;26(15):E344-7. doi:10.1097/ 00007632-200108010-00012.

5. Padua R, Padua L, Ceccarelli E, Romanini E, Zanoli $\mathrm{G}$, Bondi $\mathrm{R}$, et al. Italian version of the Roland Disability Questionnaire, specific for low-back pain: cross-cultural adaptation and validation. Eur Spine J. 2002;11(2):126-9. doi:10.1007/s005860100262.

6. The Netherlands Cancer Institute, Amsterdam. Assessing health status and quality-of-life instruments: attributes and review criteria. Qual Life Res. 2002; 11(3):193-205. doi:10.1023/A:1015291021312.

7. Ciconelli RM, Ferraz MB, Santos W, Meinão J, Quaresma MR. Tradução para a língua portuguesa e validação do questionário genérico de avaliação de qualidade de vida SF-36 (Brasil SF-36). Rev Bras Reumatol. 1999;39:143-50.

8. Ware JE Junior. Conceptualization and measurement of health related quality of life: comments on an evolving field. Arch Phys Med Rehabil. 2003;84(4 Suppl 2): S43-S51. doi:10.1053/apmr.2003.50246.

9. Dawson J, Carr A. Outcomes evaluation in orthopaedics. J Bone Joint Surg Br. 2001;83(3):313-5. doi: 10.1302/0301-620X.83B3.12148.

10. Kirkley A, Griffin S. Development of disease specific quality of life measurement tools. Arthroscpopy 2003; 19:1121-8. doi:10.1016/j.arthro.2003.10.028.
11. Amadio PC. Outcomes measurements. J Bone Joint Surg Am. 1993;75:1583-4. PMid:8245049.

12. Asher M, Min Lai S, Burton D, Manna B. The Reliability and concurrent validity of the scoliosis research society-22 patient questionnaire for idiopathic scoliosis. Spine. 2003;28(1):63-9. doi:10.1097/ 00007632-200301010-00015.

13. Asher MA, Lai SM, Glattes RC, Burton DC, Alanay A, Bago J. Refinement of the SRS-22 Health-Related Quality of Life Questionnaire function domain. Spine 2006;31:593-7. doi:10.1097/01.brs. 0000201331.50597.ea.

14. Rosanova GCL, Gabriel BS, Camarini PMF, Gianini PES, Coelho DM, Oliveira AS. Validade concorrente da versão brasileira do SRS-22r com o Br-SF-36. Rev Bras Fisioter. 2010;14(2):121-6. doi:10.1590/ S1413-35552010005000012.

15. Kapandji AI. Fisiologia articular tronco e coluna vertebral. São Paulo: Guanabara Koogan; 2001.

16. Gaudreault N, Arsenault AB, Larivière C, De Serres SJ, Rivard C-H. Assessment of the paraspinal muscles of subjects presenting an idiopathic scoliosis: an EMG pilot study. BMC Musculoskelet Disord. 2005;6:14. doi:10.1186/1471-2474-6-14.

17. Porter RW. Idiopathic Scoliosis. The Relation between the vertebral canal and the vertebral bodies. Spine. 2000;25(11):1360-6. doi:10.1097/00007632- 200006010-00007.

18. Rivett L, Rothberg A, Stewart A, Berkawitz R. The relationship between quality of life and compliance to a brace protocol in adolescents with idiopathic scoliosis: a comparative study. BMC Musculoskeletal Disorders. 2009;10:5. doi:10.1186/1471-2474-10-5.

19. Parent EC, Hill D, Moreau M, Mahood J, Raso J, Lou E. Score Distribution of the Scoliosis Quality of Life Index Questionnaire in different subgroups of patients with adolescent idiopathic scoliosis. spine. 2007;32(16):1767-77.doi:10.1097/BRS. ob013e3180b9f7a5.

20. Freidel K, Petermann F, Reichel D, Steiner A, Warschburger P, Weiss HR. Quality of life in women with idiopathic scoliosis. Spine. 2002;27(4):E87-91. doi: 10.1097/00007632-200202150-00013. 
21. Goldberg MS, Mayo NE, Poitras B, Scott S, Hanley J. The Ste-Justine Adolescent Idiopathic Scoliosis Cohort Study: Part II: perception of health, self and body image, and participation in physical activities. Spine. 1994;19(14):1562-72. doi:10.1097/ 00007632-199407001-00004.

22. Mayo NE, Goldberg MS, Poitras B, Scott S, Hanley S. The ste-Justine adolescent idiopathic scoliosis cohort study: part II: Back pain. Spine. 1994;19:1573-81. doi:10.1097/00007632-199407001-00005.

23. Payne WK 3rd, Ogilvie JW, Resnick MD, Kane RL, Transfeldt EE, Blum RW. Does scoliosis have a psychological impact and does gender make a difference? Spine. 1997;22(12):1380-4. doi:10.1097/ 00007632-199706150-00017.

24. Danielsson AJ, Wicklund I, Pehrsson K, Nickemson AL. Health related quality of life in patients with adolescent idiopathic scoliosis: a matched follow-up at least 20 years after treatment with brace or surgery. Eur Spine J. 2001;10(4):278-88. doi:10.1007/ s005860100309.

25. Danielsson AJ, Nachemson AL. Childbearing, curve progression, and sexual function in women 22 years after treatment for adolescent idiopathic scoliosis: a case-control study. Spine. 2000;26(13):1449-56. doi:10.1097/00007632-200107010-00015.

26. Danielsson AJ, Hasserius R, Onlin A, Nackemson AL. Health-related quality of life in untreated versus brace-treated patients with adolescent idiopathic scoliosis. A long term follow-up. Spine. 2010;35(2):199-205. doi:10.1097/BRS. 0b013e3181c89f4a.

27. Beaton DE, Bombardier C, Guillemin F, Ferraz MB. guidelines for the process of cross-cultural adaptation of self-report measures. Spine. 2000;25(24):3186-91. doi:10.1097/00007632-200012150-00014.
28. Chromy CA, Michael T, Carey MT, Kristopher G, Balgaard KG, Iaizzo PA. The potential use of axial spinal unloading in the treatment of adolescent idiopathic scoliosis: a case series. Arch Phys Med Rehabil. 2006;87:1447-53. doi:10.1016/j.apmr.2006.08.325.

29. Morrissy RT, Goldsmith GS, Hall EC, Kehl D, Cowie Gh. Measurement of the Cobb angle on radiographs of patients who Have Scoliosis. Evaluation of intrinsic error. J Bone Joint Surg Am. 1990;72(3):320-7. PMid:2312527.

30. Cheung KMC, Cheng EYL, Chan SCW, Yeung KWK, Luk KDK. Outcome assessment of bracing in adolescent idiopathic scoliosis by the use of the SRS-22 questionnaire. Int Orthop. 2007;31(4):507-11. doi:10.1007/ s00264-006-0209-5.

31. Wang C, Xu W, He S, Gu S, Zhao Y, Zhang J, et al. Differences in postoperative quality of life between adolescent patients with idiopathic scoliosis residing in urban and rural environments. Spine. 2010;35:652-6. doi:10.1097/BRS.0b013e3181b9fe3e.

32. Callister LC. Cultural influences on pain perceptions and behaviors. Home Health Care Manag Pract. 2003;15(3):207-11. doi:10.1177/1084822302250687.

33. Weinstein SL, Dolan LA, Cheng JC, Danielsson A, Morcuende JA. Adolescent idiopathic scoliosis. Lancet. 2008;371(9623):1527-37. doi:10.1016/ S0140-6736(08)60658-3.

Recebido: $11 / 02 / 2012$ Received: 02/11/2012

Aprovado: 09/11/2012 Approved: 11/09/2012 